\title{
Non-passivating Anion Adsorption Enables Reversible Magnesium Redox in Simple Non- Nucleophilic Electrolytes
}

Yue Sun, Qingli Zou, Wanwan Wang, and Yi-Chun Lu*

Electrochemical Energy and Interfaces Laboratory, Department of Mechanical and Automation Engineering, The Chinese University of Hong Kong, Shatin, N.T., 999077 Hong Kong, SAR, China.

\section{Corresponding Author}

*E-mail: yichunlu@mae.cuhk.edu.hk 


\section{Experimental methods}

Materials. magnesium bis(trifluoromethanesulfonyl)imide (Mg(TFSI)2, 97\%, TCI) was dried in a glass oven (Büchi) at $240{ }^{\circ} \mathrm{C}$ for $24 \mathrm{~h}$ under vacuum and stored in Ar-filled glove box (Etelux, $\mathrm{H}_{2} \mathrm{O}<1.0$ ppm, $\mathrm{O}_{2}<1.0 \mathrm{ppm}$ ) before use. Magnesium chloride $\left(\mathrm{MgCl}_{2}\right.$, anhydrous, $\geq 98 \%$ ), aluminum chloride ( $\mathrm{AlCl}_{3}, 99 \%$, anhydrous), phenyl magnesium chloride solution ( $\mathrm{PhMgCl}, 2.0 \mathrm{M}$ in tetrahydrofuran), poly(tetrafluoroethylene) (PTFE, 60\% suspension in water), 1-Methyl-2-pyrrolidinone (NMP, anhydrous, 99.5\%) and 2-propanol (anhydrous, 99.5\%) were received from Sigma-Aldrich. 1,2-Dimethoxyethane (DME, anhydrous, 99.5\%, Sigma-Aldrich) was dried with activated $4 \AA$ molecular sieves (Sigma-Aldrich) and tetrahydrofuran (THF, anhydrous, 99.9\%, Sigma-Aldrich) was dried with $3 \AA$ molecular sieves (Meryer) for one week to remove moisture. 3,4,9,10-perylenetetracarboxylic dianhydride (PTCDA, 99\%) was received from Dieckmann. Ethylenediamine (EDA) was received from XILONG Scientific. Acetone was received from RCI Labscan.

Cathode fabrication. PDI was synthesized as previous report. ${ }^{1}$ PDI was mixed with Ketjen Black EC-600JD (KB, AzkoNobel) and PTFE in IPA at the weight ratio of 5:4:1 followed by sonication for $30 \mathrm{~min}$, then the slurry was dropped on a $\Phi 12 \mathrm{~mm}$ carbon paper (AvCarb P50, AvCarb Material Solutions, for $1 \mathrm{mg} \mathrm{cm}^{-2}$ mass areal loading) and carbon felt (for $10 \mathrm{mg} \mathrm{cm}^{-2}$ mass areal loading). Finally, the electrodes were dried at $80{ }^{\circ} \mathrm{C}$ overnight to remove IPA and moisture.

Electrolyte preparation. $0.25 \mathrm{M} \mathrm{Mg}(\mathrm{TFSI})_{2}-2 \mathrm{MgCl}_{2}$ was prepared by adding $292 \mathrm{mg}$ $\mathrm{Mg}(\mathrm{TFSI})_{2}$ and $95 \mathrm{mg} \mathrm{MgCl}_{2}$ in $2 \mathrm{ml} \mathrm{DME}$ and stirring at $70{ }^{\circ} \mathrm{C}$ for half an hour. rPDI-0.25 M $\mathrm{Mg}(\mathrm{TFSI})_{2}-2 \mathrm{MgCl}_{2}$ was prepared by adding $0.8 \mathrm{mg}$ PDI $\left(0.4 \mathrm{mg} \mathrm{ml}^{-1}\right)$ in the aforementioned electrolyte and stirring with $\mathrm{Mg}$ turnings for one day until the solution turned red. The total amount of PDI added into the electrolyte is slightly larger than its solubility $(0.2 \mathrm{mM}, 0.08 \mathrm{mg}$ $\mathrm{ml}^{-1}$ ) in order to compensate its consumption during cycling.

Electrochemistry. All the two-electrode cells were assembled with a piece of $\Phi 12 \mathrm{~mm} \times 0.1$ $\mathrm{mm} \mathrm{Mg} \mathrm{disc} \mathrm{(polished} \mathrm{under} \mathrm{Ar} \mathrm{gas),} \mathrm{a} \mathrm{piece} \mathrm{of} \mathrm{quartz} \mathrm{fiber} \mathrm{(} \Phi 16 \mathrm{~mm}$, Whatman) and cathode. Quartz fibers were dried in glass oven under vacuum at $150{ }^{\circ} \mathrm{C}$ overnight before use. For $\mathrm{Mg}$ PDI full cells, a piece of $\Phi 16$ mm carbon paper was used as interlayer and PDI@carbon paper/carbon felt served as cathode. The electrolyte/cathode ratio was $140 \mathrm{ul} \mathrm{mg}^{-1}$ and $21 \mathrm{ul}$ 
$\mathrm{mg}^{-1}$ for $1 \mathrm{mg} \mathrm{cm}^{-2}$ and $10 \mathrm{mg} \mathrm{cm}^{-2}$ areal loading, respectively, and the thickness of battery components (including anode, cathode and separator) was $1.06 \mathrm{~mm}\left(1 \mathrm{mg} \mathrm{cm}^{-2}\right)$ and $1.44 \mathrm{~mm}$ $\left(10 \mathrm{mg} \mathrm{cm}^{-2}\right)$. For $\mathrm{Mg}-\mathrm{Mg}$ and $\mathrm{Mg}-\mathrm{Cu}$ cells, a piece of $\Phi 12 \mathrm{~mm} \mathrm{Mg}$ or $\mathrm{Cu}$ foil served as cathode. All the cells were assembled in glove box under Ar gas and tested with LAND Battery Testing System (Land, Wuhan Land Electronic Co., Ltd.) and VMP3 electrochemical testing unit (BioLogic, France) at $25^{\circ} \mathrm{C}$.

Materials characterizations. All the samples were washed with DME for three times with Ar protection in order to remove the residue $\mathrm{Mg}(\mathrm{TFSI})_{2}$ and $\mathrm{MgCl}_{2}$. SEM and EDS were performed on a field emission scanning electron microscopy (HR-FESEM) (FEI, Quanta 400). X-ray photoelectron spectroscopy (XPS) analysis was performed with AXIS SUPRA (Kratos Analytical Ltd.) using the mono $\mathrm{Al} \mathrm{K \alpha}$ radiation $(1486.7 \mathrm{eV})$ at the pressure lower than $5.0 \times 10^{-}$ ${ }^{9}$ Torr. The obtained XPS spectra was calibrated by $\mathrm{C} 1 \mathrm{~s}$ at $285.0 \mathrm{eV}$ and fitted with CASA.

First-principle calculations. All spin-polarization density functional theory (DFT) calculations $^{2,3}$ were carried out within the generalized gradient approximation (GGA) using the Perdew-Burke-Ernzerhof (PBE) $)^{4}$ formulation in VASP. The projected augmented wave (PAW) potentials $^{5,6}$ were chosen to describe the ionic cores and valence electrons were taken into account using a plane wave basis set with a kinetic energy cut off of $400 \mathrm{eV}$. Partial occupancies of the Kohn-Sham orbitals were allowed with the Gaussian smearing method and a width of $0.05 \mathrm{eV}$. The electronic energy was considered to reach self-consistence when the energy change was smaller than $10^{-6} \mathrm{eV}$. A geometry optimization was considered convergent when the energy change was smaller than $0.05 \mathrm{eV} \AA^{-1}$. The vacuum spacing in a direction perpendicular to the plane of the structure is $15 \AA$. The Brillouin zone integration was performed using $3 \times 3 \times 1$ Monkhorst-Pack k-point sampling for a structure. Finally, the adsorption energies (Eads) were calculated as $E_{a d s}=E_{a d} /$ sub $-E_{a d}-E_{s u b}$, where $E_{a d} /$ sub, $E_{a d}$, and $E_{\text {sub }}$ are the energies of the optimized adsorbate/substrate system, the adsorbate and the clean substrate, respectively. 

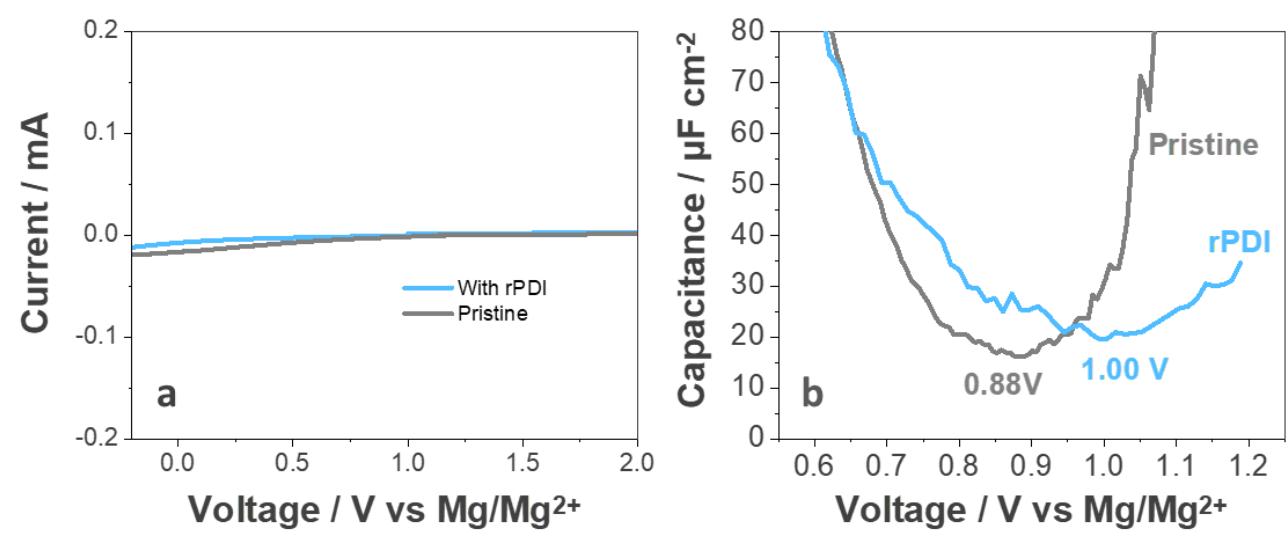

Figure S1. (a) Linear sweep voltammetry of $\mathrm{Mg}-\mathrm{Cu}$ cells with rPDI electrolyte (blue line) and pristine electrolyte (gray line). (b) Capacitance-potential profile of $\mathrm{Mg}-\mathrm{Cu}$ cells with rPDI electrolyte (blue line) and pristine electrolyte (gray line). Alternating current voltammetry (ACV) was conducted between $0.6-1.2 \mathrm{~V}$ where non-faradic process occurred (Figure S1a).

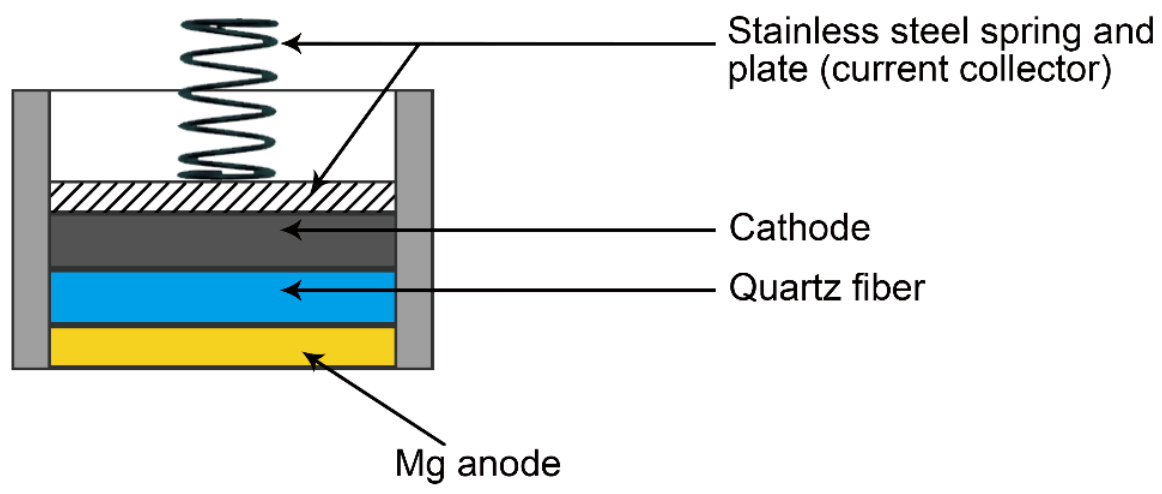

Figure S2. Cell configuration for galvanostatic test. 


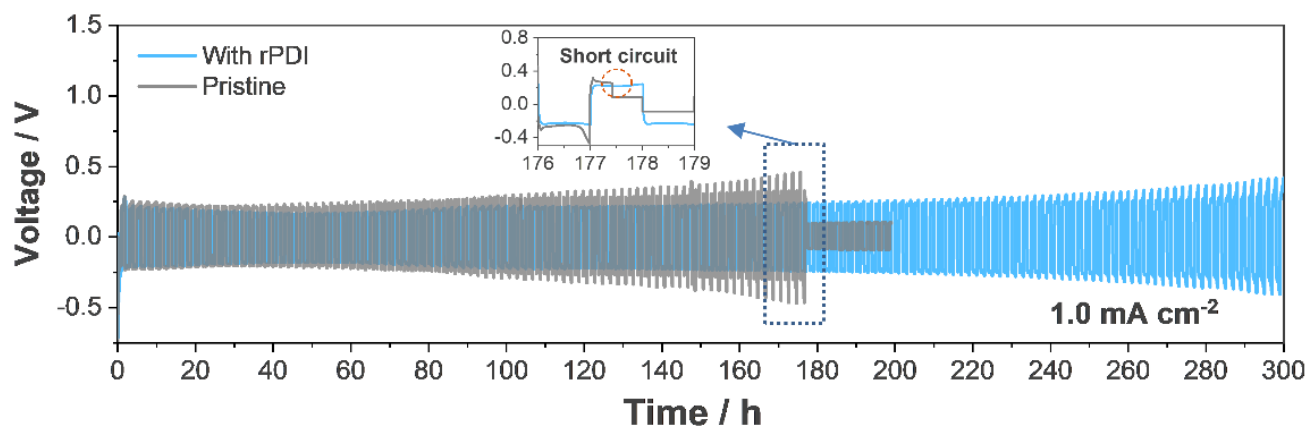

Figure S3. Cycling stability of Mg-Mg symmetric cells with pristine and rPDI electrolyte at $1.0 \mathrm{~mA} \mathrm{~cm}{ }^{-2}$. The areal capacity was $1.0 \mathrm{mAh} \mathrm{cm}^{-2}$ for each charge/discharge process.
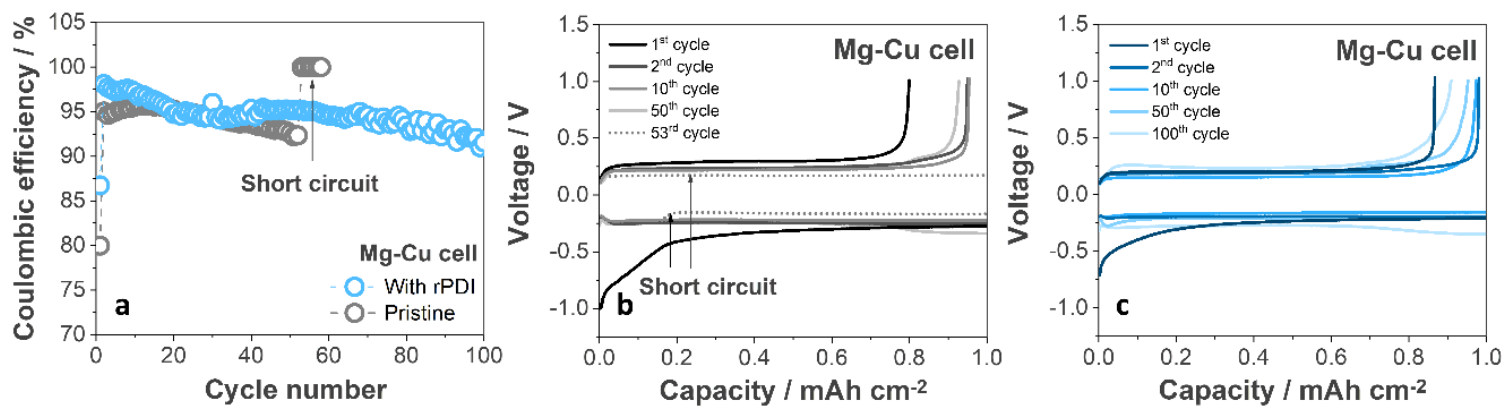

Figure S4. a) Coulombic efficiency of $\mathrm{Mg}-\mathrm{Cu}$ cells with pristine and rPDI electrolyte at 1.0 $\mathrm{mA} \mathrm{cm}{ }^{-2}$. Galvanostatic voltage profile of $\mathrm{Mg}-\mathrm{Cu}$ cells at $1.0 \mathrm{~mA} \mathrm{~cm}^{-2}$ with $\mathrm{b}$ ) pristine and c) rPDI electrolyte. 

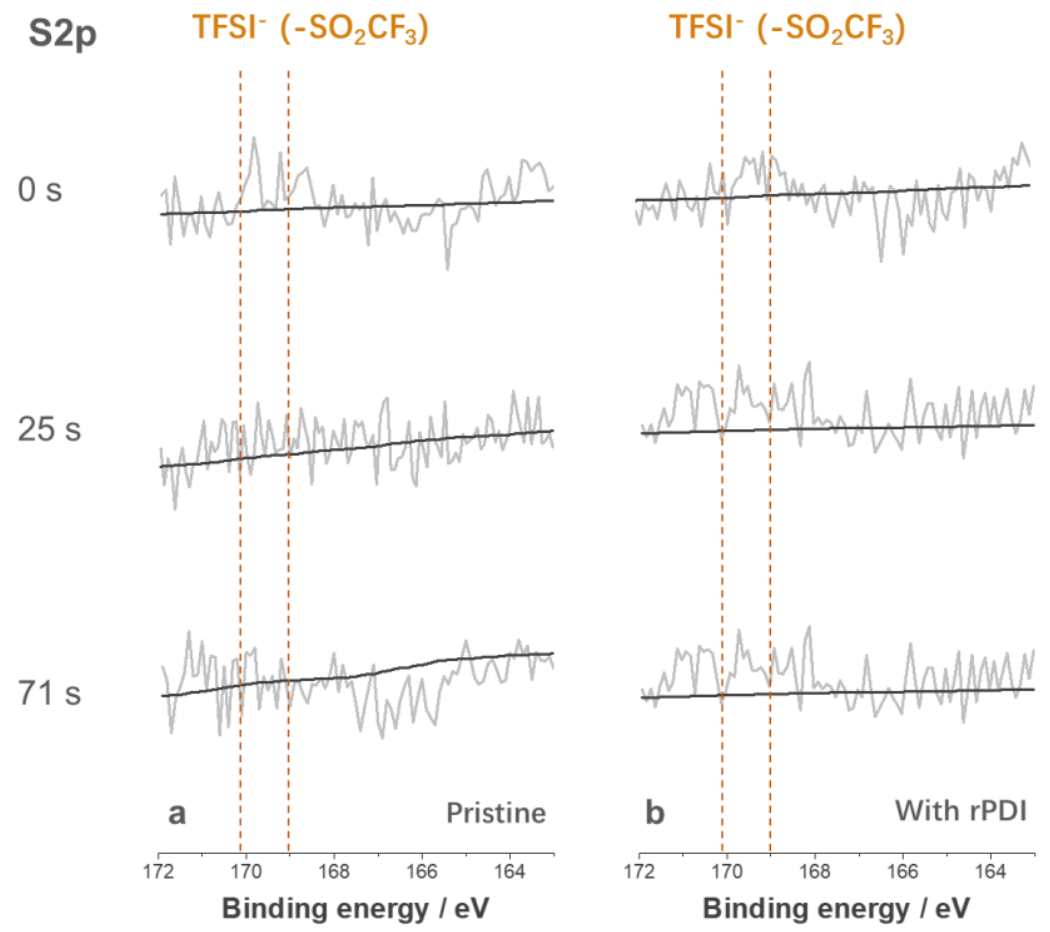

Figure S5. S2p XPS spectrum of the Mg electrode cycled a) with pristine electrolyte and b) with rPDI electrolyte.
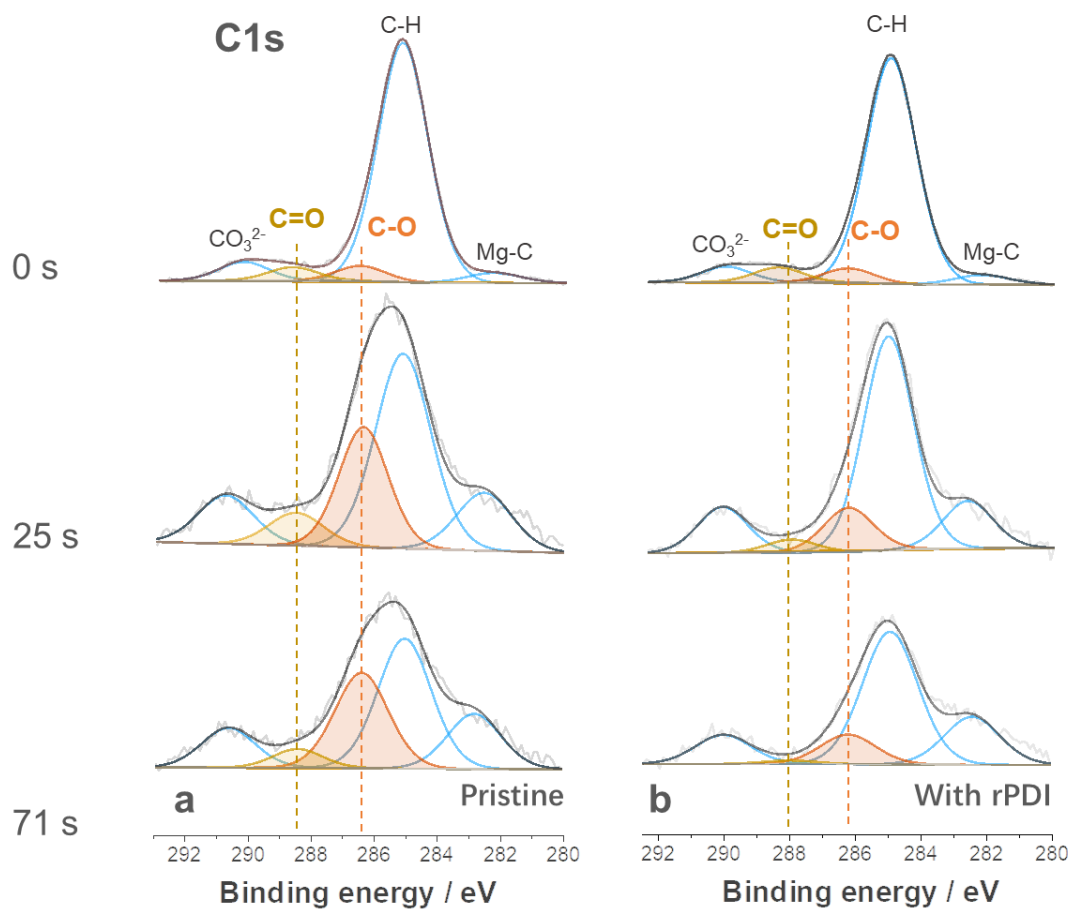

Figure S6. C1s XPS spectrum of the Mg electrode cycled a) with pristine electrolyte and b) with rPDI electrolyte. 

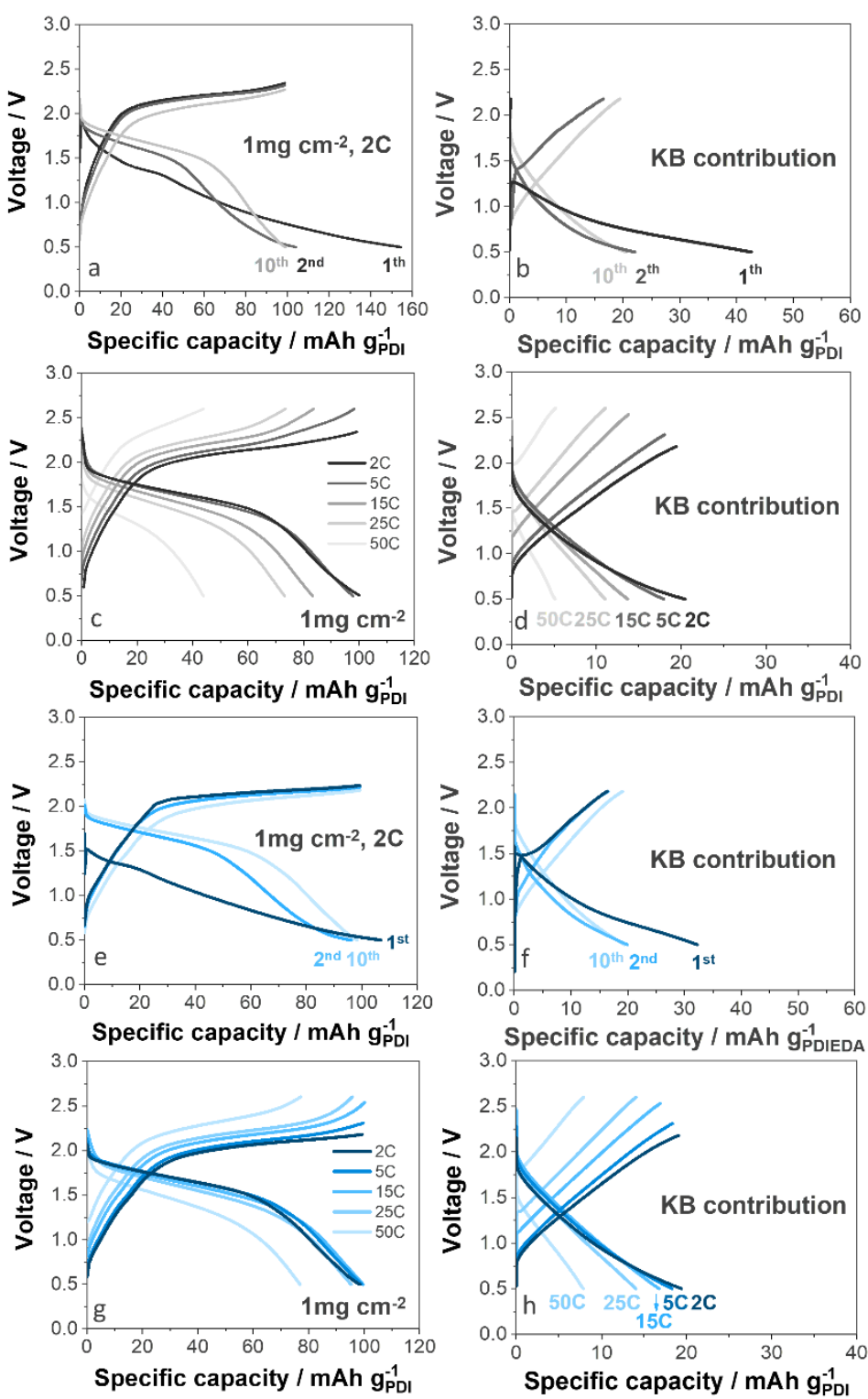

Figure S7. Galvanostatic profile of a) $1.0 \mathrm{mg} \mathrm{cm}^{-2} \mathrm{PDI} / 0.8 \mathrm{mg} \mathrm{cm}^{-2}$ Ketjen black (KB) at $2 \mathrm{C}$ between $1^{\text {st }}-10^{\text {th }}$ cycles in pristine electrolyte; b) $0.8 \mathrm{mg} \mathrm{cm} \mathrm{cm}^{-2} \mathrm{~KB}$ at $2 \mathrm{C}$ between $1^{\text {st }}-10^{\text {th }}$ cycles in pristine electrolyte; c) $1.0 \mathrm{mg} \mathrm{cm}^{-2} \mathrm{PDI} / 0.8 \mathrm{mg} \mathrm{cm}^{-2} \mathrm{~KB}$ at $2 \mathrm{C}-50 \mathrm{C}$ in pristine electrolyte; d) $0.8 \mathrm{mg} \mathrm{cm}^{-2} \mathrm{~KB}$ at $2 \mathrm{C}-50 \mathrm{C}$ in pristine electrolyte; e) $1.0 \mathrm{mg} \mathrm{cm} \mathrm{cm}^{-2} \mathrm{PDI} / 0.8$ $\mathrm{mg} \mathrm{cm} \mathrm{cm}^{-2} \mathrm{~KB}$ at $2 \mathrm{C}$ between $1^{\text {st }}-10^{\text {th }}$ cycles in rPDI electrolyte; f) $0.8 \mathrm{mg} \mathrm{cm}^{-2} \mathrm{~KB}$ at $2 \mathrm{C}$ between $1^{\text {st }}-10^{\text {th }}$ cycles in rPDI electrolyte; g) $1.0 \mathrm{mg} \mathrm{cm}^{-2} \mathrm{PDI} / 0.8 \mathrm{mg} \mathrm{cm}^{-2} \mathrm{~KB}$ at $2 \mathrm{C}-50 \mathrm{C}$ in rPDI electrolyte; h) $0.8 \mathrm{mg} \mathrm{cm}^{-2} \mathrm{~KB}$ at $2 \mathrm{C}-50 \mathrm{C}$ in rPDI electrolyte; All the specific capacities and current densities were calculated based on the loading and theoretical capacity of PDI (1.0 mg cm $\left.\mathrm{mg}^{-2}, 128 \mathrm{mAh} \mathrm{g}^{-1}\right)$. 


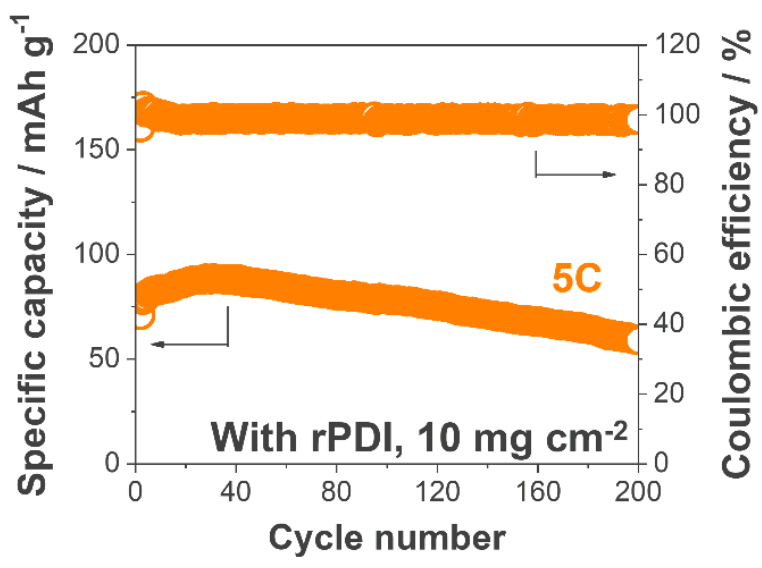

Figure S8. Cycling stability of Mg-PDI full cells with rPDI electrolyte at $5 \mathrm{C}$ under $10 \mathrm{mg} \mathrm{cm}^{-}$ ${ }^{2}, 1 \mathrm{C}=128 \mathrm{~mA} \mathrm{~g}^{-1}$.
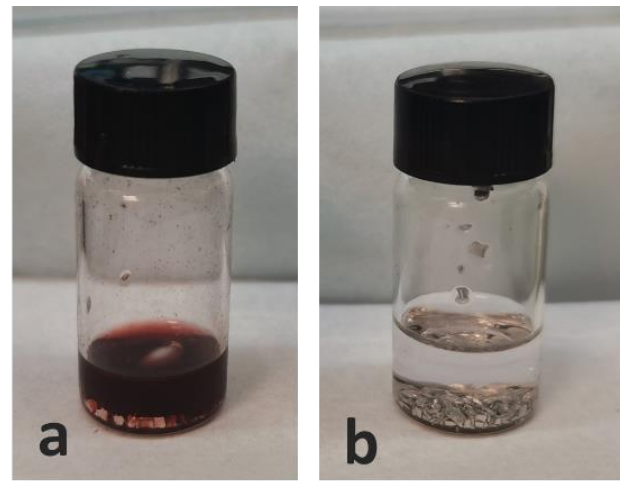

Figure S9. PDI a) stirred for one day and b) rested with $\mathrm{Mg}$ turnings in $0.25 \mathrm{M} \mathrm{Mg}(\mathrm{TFSI})_{2}-$ $2 \mathrm{MgCl}_{2} / \mathrm{DME}$. After stirring, the solution turned deep red, indicating the formation of rPDI, while that without stirring was colorless. 
Table S1. Summary of electrochemical performance of state-of-the-art magnesium batteries

\begin{tabular}{|c|c|c|c|c|c|}
\hline & System & Electrolyte & $\begin{array}{c}\text { Areal } \\
\text { power } \\
\text { density } \\
{\left[\mu \mathrm{W} \mathrm{cm}^{-2}\right]}\end{array}$ & $\begin{array}{c}\text { Areal } \\
\text { capacity } \\
{\left[\mu \mathrm{Ah} \mathbf{c m}^{-2}\right]}\end{array}$ & $\begin{array}{l}\text { Cycling } \\
\text { retention }\end{array}$ \\
\hline \multirow{5}{*}{$\begin{array}{l}\text { Mg(TFSI) } 2 \\
\text { based } \\
\text { electrolytes }\end{array}$} & \multirow{2}{*}{$\begin{array}{l}\text { Mg/PDI, } \\
\text { this work }\end{array}$} & \multirow{2}{*}{$\begin{array}{c}\mathrm{rPDI}+0.25 \mathrm{M} \\
\mathrm{Mg}(\mathrm{TFSI})_{2}-2 \mathrm{MgCl}_{2} \\
\text { in DME }\end{array}$} & 2007 & 1064 & $\begin{array}{c}92.1 \% \text { retention } \\
\text { after } 200 \text { cycles } \\
\text { at } 1 \mathrm{C} \\
\end{array}$ \\
\hline & & & 8960 & 882 & $\begin{array}{c}83.1 \% \text { retention } \\
\text { after } 200 \text { cycles } \\
\text { at } 5 \mathrm{C} \\
\end{array}$ \\
\hline & $\mathrm{Mg} / \mathrm{COF}^{7}$ & $\begin{array}{c}0.5 \mathrm{M} \mathrm{Mg}(\mathrm{TFSI})_{2} \text { in } \\
\mathrm{DME}\end{array}$ & 1226 & 108 & $\begin{array}{c}51.4 \% \text { retention } \\
\text { after } 3000 \text { cycles } \\
\text { at } 5 \mathrm{C} \\
\end{array}$ \\
\hline & $\mathrm{Mg} / \mathrm{NP}^{8}$ & $\begin{array}{c}0.4 \mathrm{M} \mathrm{Mg}(\mathrm{TFSI})_{2-} \\
\mathrm{MgCl}_{2} \text { in } \\
\text { TEGDME/DOL }\end{array}$ & 320 & 342 & $\begin{array}{c}76.5 \% \text { retention } \\
\text { after } 50 \text { cycles } \\
\text { at } 0.25 \mathrm{C}\end{array}$ \\
\hline & $\begin{array}{l}\mathrm{Mg} / \mathrm{P}(\mathrm{NDI} 2 \mathrm{O} \\
-\mathrm{D}-\mathrm{T} 2)^{9}\end{array}$ & $\begin{array}{c}0.2 \mathrm{M} \mathrm{Mg}(\mathrm{TFSI})_{2} \text { in } \\
\text { diglyme }\end{array}$ & 394 & 48 & $\begin{array}{c}87.5 \% \text { retention } \\
\text { after } 2500 \text { cycles } \\
\text { at } 5.6 \mathrm{C} \\
\end{array}$ \\
\hline \multirow{3}{*}{$\begin{array}{c}\text { Organochloro } \\
\text {-aluminates } \\
\text { based } \\
\text { electrolytes }\end{array}$} & $\begin{array}{c}\mathrm{Mg} / \\
\text { PI2@CNTs }{ }^{10}\end{array}$ & $\begin{array}{c}0.25 \mathrm{M} \\
\mathrm{Mg}\left(\mathrm{HMDS}_{2^{-}}\right. \\
4 \mathrm{MgCl}_{2} / \\
\text { 2THF-PP14TFSI }\end{array}$ & 4740 & 80 & $\begin{array}{c}52.5 \% \text { retention } \\
\text { after } 8000 \text { cycles } \\
\text { at } 20 \mathrm{C}\end{array}$ \\
\hline & $\mathrm{Mg} / \mathrm{I}_{2}{ }^{11}$ & $\begin{array}{c}0.5 \mathrm{M} \mathrm{Mg}(\mathrm{HMDS})_{2} \\
-\mathrm{AlCl}_{3}-\mathrm{MgCl}_{2} \text { in } \\
\mathrm{TEGDME}\end{array}$ & 224 & 175 & $\begin{array}{c}94.6 \% \text { retention } \\
\text { after } 120 \text { cycles } \\
\text { at } 0.5 \mathrm{C} \\
\end{array}$ \\
\hline & $\mathrm{Mg} / 14 \mathrm{PAQ}^{12}$ & $\begin{array}{c}0.3 \mathrm{M} \mathrm{Mg}(\mathrm{HMDS})_{2^{-}} \\
4 \mathrm{MgCl}_{2} / \mathrm{THF}\end{array}$ & 585 & 131 & $\begin{array}{c}90.4 \% \text { retention } \\
\text { after } 1000 \text { cycles } \\
\text { at } 1 \mathrm{C} \\
\end{array}$ \\
\hline \multirow{2}{*}{$\begin{array}{l}\text { Carborane } \\
\text { based } \\
\text { electrolyte }\end{array}$} & \multirow{2}{*}{$\mathrm{Mg} / \mathrm{PTO}^{13}$} & \multirow{2}{*}{$\begin{array}{c}0.5 \mathrm{M}\left[\mathrm{Mg}(\mathrm{DME})_{3}\right] \\
\left(\mathrm{CB}_{11} \mathrm{H}_{12}\right)_{2} \text { in } \\
\text { DME/Diglyme }\end{array}$} & 363 & 788 & $\begin{array}{c}84.0 \% \text { retention } \\
\text { after } 500 \text { cycles } \\
\text { at } 0.2 \mathrm{C}\end{array}$ \\
\hline & & & 9190 & 728 & $\begin{array}{c}82.0 \% \text { retention } \\
\text { after } 200 \text { cycles } \\
\text { at } 5 \mathrm{C}\end{array}$ \\
\hline
\end{tabular}




\section{References}

(1) Lu, D.; Liu, H.; Huang, T.; Xu, Z.; Ma, L.; Yang, P.; Qiang, P.; Zhang, F.; Wu, D. Magnesium Ion Based Organic Secondary Batteries. J. Mater. Chem. A 2018, 6 (36), 17297-17302. DOI: 10.1039/C8TA05230A.

(2) Kresse, G.; Furthmüller, J. Efficiency of Ab-Initio Total Energy Calculations for Metals and Semiconductors Using a Plane-Wave Basis Set. Computational materials science 1996, 6 (1), 15-50.

(3) Kresse, G.; Furthmüller, J. Efficient Iterative Schemes for Ab Initio Total-Energy Calculations Using a Plane-Wave Basis Set. Physical review B 1996, 54 (16), 11169.

(4) Perdew, J. P.; Burke, K.; Ernzerhof, M. Generalized Gradient Approximation Made Simple. Physical review letters 1996, 77 (18), 3865.

(5) Kresse, G.; Joubert, D. From Ultrasoft Pseudopotentials to the Projector AugmentedWave Method. Physical review b 1999, 59 (3), 1758.

(6) Blöchl, P. E. Projector Augmented-Wave Method. Physical review B 1994, 50 (24), 17953.

(7) Sun, R.; Hou, S.; Luo, C.; Ji, X.; Wang, L.; Mai, L.; Wang, C. A Covalent Organic Framework for Fast-Charge and Durable Rechargeable Mg Storage. Nano Lett. 2020, 20 (5), 3880-3888. DOI: 10.1021/acs.nanolett.0c01040.

(8) Bančič, T.; Bitenc, J.; Pirnat, K.; Kopač Lautar, A.; Grdadolnik, J.; Randon Vitanova, A.; Dominko, R. Electrochemical Performance and Redox Mechanism of NaphthaleneHydrazine Diimide Polymer as a Cathode in Magnesium Battery. Journal of Power Sources 2018, 395, 25-30. DOI: 10.1016/j.jpowsour.2018.05.051.

(9) Dong, H.; Liang, Y.; Tutusaus, O.; Mohtadi, R.; Zhang, Y.; Hao, F.; Yao, Y. Directing Mg-Storage Chemistry in Organic Polymers toward High-Energy Mg Batteries. Joule 2019, 3 (3), 782-793. DOI: 10.1016/j.joule.2018.11.022.

(10) Wang, Y.; Liu, Z.; Wang, C.; Hu, Y.; Lin, H.; Kong, W.; Ma, J.; Jin, Z. $\pi$-Conjugated Polyimide-Based Organic Cathodes with Extremely-Long Cycling Life for Rechargeable Magnesium Batteries. Energy Storage Materials 2020, 26, 494-502. DOI: 10.1016/j.ensm.2019.11.023.

(11) Tian, H.; Gao, T.; Li, X.; Wang, X.; Luo, C.; Fan, X.; Yang, C.; Suo, L.; Ma, Z.; Han, W.; Wang, C. High Power Rechargeable Magnesium/Iodine Battery Chemistry. Nature Communications 2017, 8, 14083. DOI: 10.1038/ncomms 14083.

(12) Pan, B.; Huang, J.; Feng, Z.; Zeng, L.; He, M.; Zhang, L.; Vaughey, J. T.; Bedzyk, M. J.; Fenter, P.; Zhang, Z.; Burrell, A. K.; Liao, C. Polyanthraquinone-Based Organic Cathode for High-Performance Rechargeable Magnesium-Ion Batteries. Advanced Energy Materials 2016, 6 (14), 1600140. DOI: 10.1002/aenm.201600140.

(13) Dong, H.; Tutusaus, O.; Liang, Y.; Zhang, Y.; Lebens-Higgins, Z.; Yang, W.; Mohtadi, R.; Yao, Y. High-Power Mg Batteries Enabled by Heterogeneous Enolization Redox Chemistry and Weakly Coordinating Electrolytes. Nat Energy 2020, 5, 1043-1050. DOI: 10.1038/s41560-020-00734-0. 